\title{
Effect of Hetero Atom on the Hammett's Reaction Constant $(\rho)$ from the Physical Basis of Dissociation Equilibriums of (Dithio) Benzoic Acids and (Thio) Phenols and Its Application to Solvolysis Reactions and Some Free Radical Reactions
}

\author{
Jagannadham Vandanapu ${ }^{1}$ and Sanjeev Rachuru ${ }^{2}$ \\ ${ }^{1}$ Department of Chemistry, Osmania University, Hyderabad 500 007, India \\ ${ }^{2}$ Department of Chemistry, Mizan-Tepi University, Tepi Campus, Tepi, Ethiopia \\ Correspondence should be addressed to Jagannadham Vandanapu, jagannadham1950@yahoo.com \\ Received 3 April 2012; Accepted 11 May 2012 \\ Academic Editor: Leonardo Palmisano
}

Copyright (C 2012 J. Vandanapu and S. Rachuru. This is an open access article distributed under the Creative Commons Attribution License, which permits unrestricted use, distribution, and reproduction in any medium, provided the original work is properly cited.

\begin{abstract}
The emergence of putative Hammett equation in mid 1930s was a boon to physical-organic chemists to elucidate the reaction mechanisms of several organic reactions. Based on the concept of this equation several hundreds of papers have emerged in chemical literature in the last century on the effect of structure, on reactivity, and very few on thermodynamic stability and kinetic reactivity of intermediates. In this article an attempt is made to explain the effect of hetero atom on Hammett's reaction constant $(\rho)$ taking the dissociation equilibriums of benzoic acids, dithiobenzoic acids, phenols, and thiophenols.
\end{abstract}

\section{Introduction}

Ever since the Hammett equation was developed $[1,2]$, there were several hundreds of redox, condensation, disproportionation, nucleophilic and electrophilic substitution, and addition reactions with meta- and para-substituted benzene derivatives in the literature, for which the Hammett reaction $(\rho)$ constants were reported. Inclusion of those references here is beyond the scope this article as they run into several pages. However the readers can find many articles and reviews in several standard physical-organic chemistry text books. In addition to these numerous reactions, a few reactions were reported by one of the authors (V. Jaganndham) from elsewhere [3] and from our laboratory $[4,5]$ on the solvolysis and reactions of intermediate carbocations with nucleophilic solvent water. An effect of $\alpha$-hetero atom substitution on kinetic and thermodynamic stability of intermediate carbocations were also reported from elsewhere $[6,7]$ and from our laboratory [8]. But in these reactions [3-5] no attempt is made to explain the effect of $\alpha$-hetero atom on the Hammett reaction constant $(\rho)$, which we tried to explain in the present work taking the title equilibriums as staple examples.

\section{Results and Discussion}

The effect of substituent either in meta- or para-position in the benzene ring on the rate or equilibrium constant is given by Hammett [2] in the form of a formula:

$$
-R T \ln K+R T \ln K^{\circ}=\Delta F=\frac{A}{d^{2}}\left(\frac{B_{1}}{D}+B_{2}\right),
$$

where $K$ is the equilibrium or rate constant of the substituted reactant, $K^{\circ}$ is that of unsubstituted reactant, " $\Delta F$ " is the free energy change for equilibrium process or rate process, " $d$ " is the distance between the substituent and the reaction center, " $D$ " is the dielectric constant of the medium, and $A, B_{1}$, and $B_{2}$ are the constants. Here $A$ depends on the substituent and $B_{1}$ and $B_{2}$ depend on the nature of the reaction. Later, based 


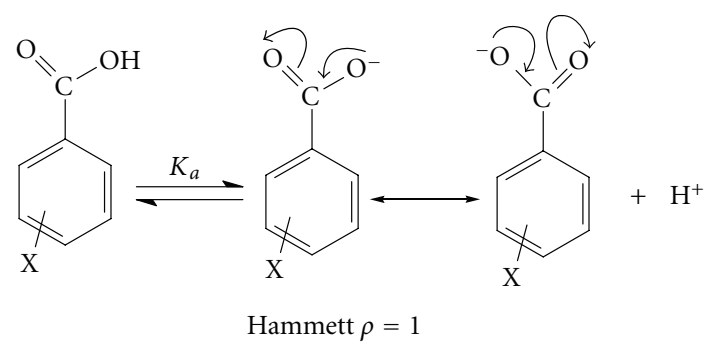

SCHEMe 1

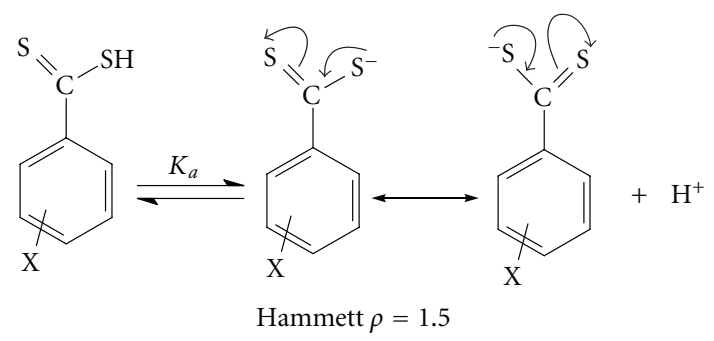

SCHEMe 2

on some experimental observations Hammett rearranged (1) to the form:

$$
\log K=\log K^{\circ}+\rho \sigma,
$$

where $\sigma=-A / 2.303 R$ and $\rho=\left(1 / d^{2} T\right)\left(B_{1} / D+B_{2}\right),(2)$ is now known as famous Hammett equation. The magnitude of $\sigma$ depends on the substituent and $\rho$ depends on the nature of the reaction, medium, and temperature. Now the question is the evaluation of Hammett substituent constant $(\sigma)$. For this the values of $K, K^{\circ}$, and Hammett reaction constant $(\rho)$ are needed. $K$ and $K^{\circ}$ are experimentally determinable quantities. Therefore the choice of a value of unity for the reaction constant $(\rho)$ in the ionization equilibriums of substituted benzoic acids in water solution at $25^{\circ} \mathrm{C}$ was determined by the large amount of accurate data available from the work of Dippy and his coworkers [9-11]. With the core of $\sigma$ values thus obtained, the Hammett reaction constants $(\rho)$ were obtained for several other reactions. Thus in turn using the Hammett reaction constant $(\rho)$, the unknown or accurately not known Hammett substituent constants $(\rho)$ were determined for other substituents. In conclusion it is understood from the Hammett's work on effect of substituents on reaction equilibriums and rates, that the reaction constant $(\rho)$ is one for the dissociation of benzoic acids in aqueous solution at $25^{\circ} \mathrm{C}$ (Scheme 1).

When the hetero atom is changed from oxygen to sulfur that is for the dissociation of dithiobenzoic acids the Hammett reaction constant $(\rho)$ was found to be 1.5 (Scheme 2).

A plot of $\mathrm{p} K_{a}$ [12] versus Hammett substituent constant $(\sigma)$ was excellently linear with a slope of 1.5 and correlation coefficient of 0.9964 (Figure 1).

The reasons for high Hammett reaction constant $(\rho)$ are:

The Hammett reaction constant $(\rho)$ for dithiobenzoic acids dissociation equilibriums is one and half times greater

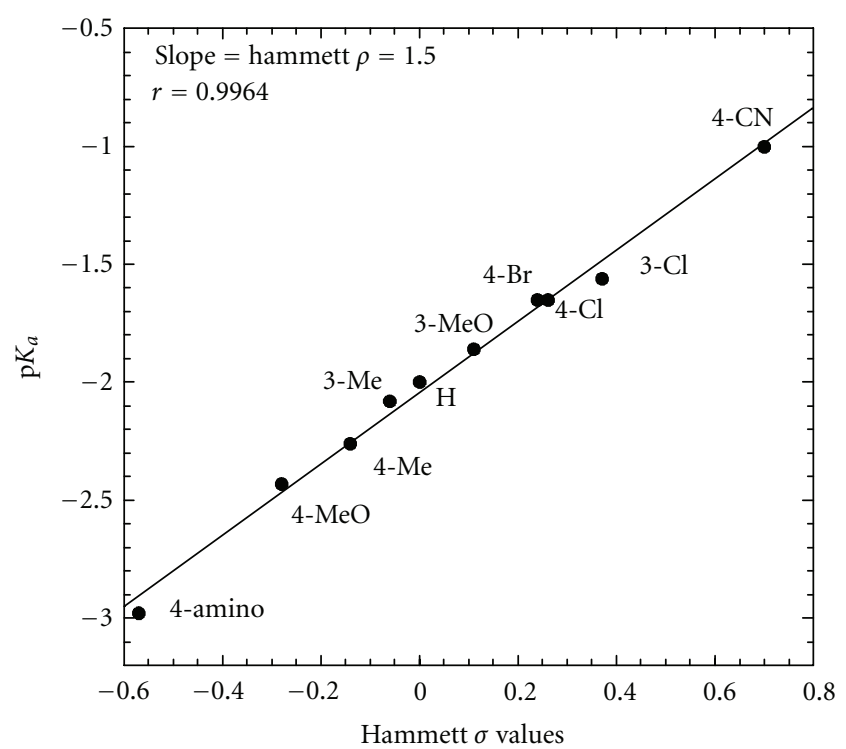

Figure 1: Plot of $\mathrm{p} K_{a}$ values versus Hammett sigma values for dithiobenzoic acid dissociation equilibriums.

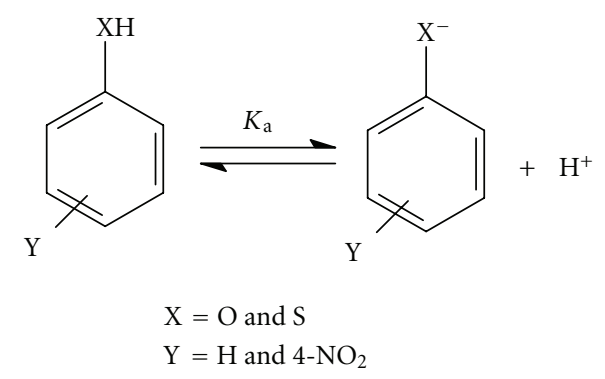

Hammett reaction constant $=2.5$

when $\mathrm{X}=\mathrm{S}$

and it is 2.1 when $\mathrm{X}=\mathrm{O}$

Scheme 3

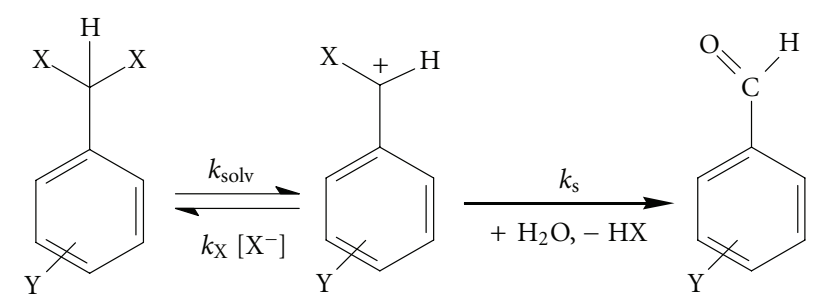

When $\mathrm{X}=\mathrm{Cl}$

Hammett's $\rho$ for solvolysis step is -2.05

for water reaction it is 1.17

When $\mathrm{X}=\mathrm{Br}$

Hammett's $\rho$ for solvolysis step is -5.49

for water reaction it is 3.74

SCHEme 4 


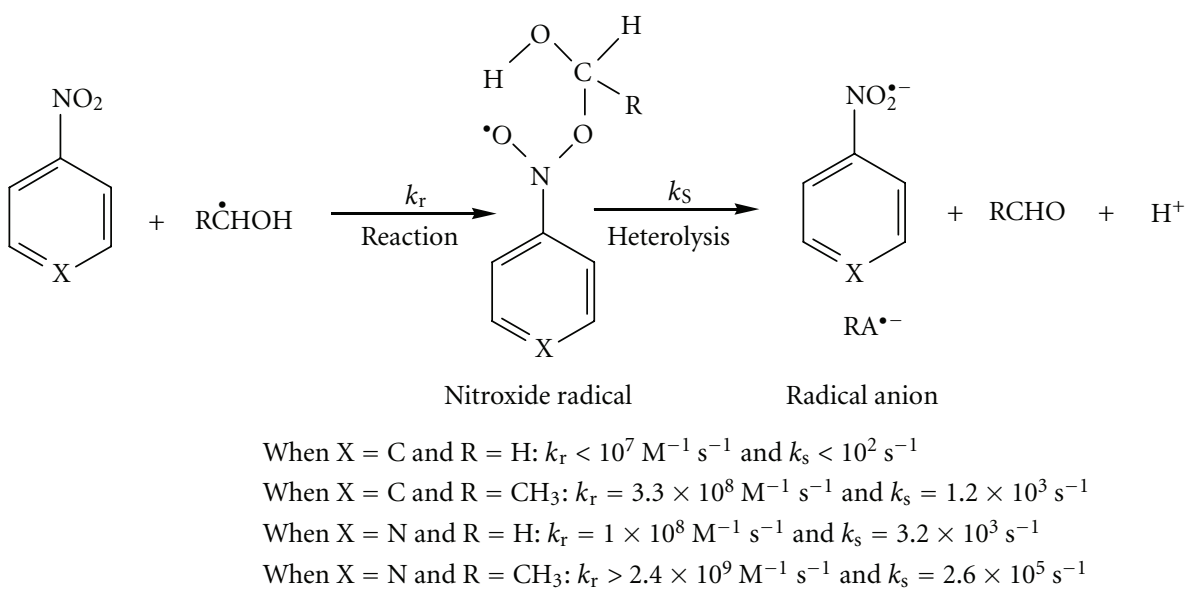

Scheme 5

than that of benzoic acids dissociation equilibriums. The magnitude of $\rho$ depends on several factors like stability of transition state. Since the $\rho$ value of dithiobenzoic acid is greater than that of benzoic acid dissociation equilibrium series, an implicit conclusion is that the transition state is far more stable than the transition state of benzoic acid dissociation equilibrium series. This is tacitly comprehensible from the ease with which sulfur can involve its lone pair of electrons in resonance than the ease with which oxygen can involve its lone pair of electrons (sulfur $3 \mathrm{~s}^{2} 3 \mathrm{p}^{4}$ and oxygen $\left.2 \mathrm{~s}^{2} 2 \mathrm{p}^{4}\right)$. Here we are referring to resonance in $\mathrm{S}=\mathrm{C}-\mathrm{S}^{-} \leftrightarrow \mathrm{S}^{-}-$ $\mathrm{C}=\mathrm{S}$ that is far more pronounced than $\mathrm{O}=\mathrm{C}-\mathrm{O}^{-}{ }^{-} \mathrm{O}-\mathrm{C}=\mathrm{O}$ because of the relative ease with which sulfur can donate its lone pair of electrons than oxygen. In general, sulfur is very nucleophilic because of its large size, which makes it readily polarizable, and its lone pairs of electrons are readily accessible. The same observations were made in the study of kinetic and thermodynamic stability of $\alpha$-oxygen- and $\alpha$ sulfur-stabilized carbocations in solution $[6,7]$.

Similarly the Hammett reaction constant $(\rho)$ for thiophenol dissociation equilibriums was computed from the $\mathrm{p} K_{a}$ [12] values of thiophenol and 4-nitro thiophenol dissociation equilibriums and it came out to be 2.5 (Scheme 3 ).

Though this value is only from two thiophenols, the trend in the increase in Hammett reaction constant $(\rho)$ is unmistakable. And this is about 20\% higher than the Hammett reaction constant $(\rho=2.1)$ of phenol dissociation equilibriums [13]. The same reasons offered above for the dissociation equilibriums of dithiobenzoic acids also hold well here.

Hence it is very clear that the effect of hetero atom on the benzoic acid/phenol dissociation equilibriums is pretty promising and in turn on Hammett's reaction constant $(\rho)$.

Another notable and interesting observation of the effect of hetero atom in kinetics is in the study of solvolysis reactions ofbenzal halides $[4,5]$. In these studies the authors have found out a marked influence of effect of chlorine and bromine atoms on the formation $\left(k_{\text {solv }}\right)$ of $\alpha$-chloro and $\alpha$ bromobenzyl carbocations from their corresponding neutral gem-adducts and on the reaction of the cations $\left(k_{s}\right)$ with nucleophilic water. The Hammett reaction constant $\left(\rho_{\text {solv }}\right)$ for $k_{\text {solv }}$ step in the solvolysis of gem-dichlorides is -2.05 [4]. The same $\left(\rho_{s}\right)$ for addition of water to the cation is 1.17 [4], while in gem-dibromide reactions $\rho_{\text {solv }}$ is -5.49 and $\rho_{s}$ is 3.74 [5]. Therefore it is very clear that there was a threetime increase in Hammett's $\rho$ value in the formation of the cations when we move the hetero atom from chlorine to bromine and for the reaction of the cation with water the increase is about three and half times in the Hammett $\rho$ value (Scheme 4).

These Hammett reaction constants depend largely on the stabilities of the intermediate $\alpha$-chloro and $\alpha$-bromobenzyl carbocations, that is, their formation from neutral halide ion adducts and their reaction with nucleophile (water). The intrinsic barrier for capture of resonance stabilized carbocations by nucleophiles results largely from loss of resonance interactions in the transition state by bond formation to the nucleophile. The lower intrinsic barrier for formation and larger intrinsic barrier for capture of $\alpha$-bromobenzyl carbocations by solvent water (than of $\alpha$ chloro stabilized benzyl carbocations) was consistent with the fact that the $\alpha$-bromo substituted benzyl carbocations were more stable than those of their chloro analogs. This was due to more polarizability and larger size of bromo substituent, as compared to the chloro substituent. Hence, the Hammett $\rho$ values for the both processes were higher for bromobenzyl carbocations.

From one of the author's work published from elsewhere [14], now it is the turn for " $N$ " when it replaces " $C$ " on some free radical reactions, though it is not the effect of hetero atom on Hammett's $\rho$ value but on the effect of hetero atom on the formation reaction $\left(k_{r}\right)$ of nitroxide radical and its heterolysis $\left(k_{s}\right)$ is still quite stanch and striking. The example of these reactions is the reactions of nitrobenzene and 4-nitropyridine with $\alpha$-hydroxy methyl and $\alpha$-hydroxy ethyl radicals. Due to electron-withdrawing effect of the nitrogen in the ring, and whose electronegativity (3.0) is greater than that of carbon (2.5), 4-nitropyridine is 
much more electron deficient than nitrobenzene and even nitrobenzenes carrying electron-withdrawing substituents such as $\mathrm{NO}_{2}$. This is reflected by the one-electron reduction potentials $[15,16]$ of nitro compounds. With ${ }^{\bullet} \mathrm{CH}_{2} \mathrm{OH}, 4$ nitropyridine forms an adduct (nitroxide radical) with $k_{r}=$ $1.0 \times 10^{8} \mathrm{M}^{-1} \mathrm{~s}^{-1}$ to be compared with $<10^{7} \mathrm{M}^{-1} \mathrm{~s}^{-1}$ for the case of nitrobenzene, which undergoes heterolysis to give nitro compound radical anion $\left(\mathrm{RA}^{{ }^{-}}\right)$, formaldehyde, and $\mathrm{H}^{+}$, and with $k_{s}=3.2 \times 10^{3} \mathrm{~s}^{-1}$ to be compared with $<10^{2} \mathrm{~s}^{-1}$ for the case of nitrobenzene. With $\mathrm{CH}_{3}{ }^{\circ} \mathrm{CHOH}, 4-$ nitropyridine forms an adduct (nitroxide radical) with $k_{r}>$ $2.4 \times 10^{9} \mathrm{M}^{-1} \mathrm{~s}^{-1}$ to be compared with $3.3 \times 10^{8} \mathrm{M}^{-1} \mathrm{~s}^{-1}$ for the case of nitrobenzene which undergoes heterolysis to acetaldehyde, nitro compound radical anion $\left(\mathrm{RA}^{\bullet-}\right)$, and $\mathrm{H}^{+}$ and with $k_{s}=2.6 \times 10^{5} \mathrm{~s}^{-1}$ to be compared with $1.2 \times 10^{3} \mathrm{~s}^{-1}$ for the case of nitrobenzene. All this has been shown in the Scheme 5.

\section{References}

[1] L. P. Hammett, "Some relations between reaction rates and equilibrium constants," Chemical Reviews, vol. 17, no. 1, pp. 125-136, 1935.

[2] L. P. Hammett, "The effect of structure upon the reactions of organic compounds. Benzene derivatives," Journal of the American Chemical Society, vol. 59, no. 1, pp. 96-103, 1937.

[3] J. P. Richard, T. L. Amyes, V. Jagannadham, Y. G. Lee, and D. J. Rice, "Spontaneous cleavage of gem-diazides: a comparison of the effects of $\alpha$-azido and other electron-donating groups on the kinetic and thermodynamic stability of benzyl and alkyl carbocations in aqueous solution," Journal of the American Chemical Society, vol. 117, no. 19, pp. 5198-5205, 1995.

[4] R. Sanjeev and V. Jagannadham, "Substituent effects on the spontaneous cleavage of benzyl-gem-dichlorides in aqueous solution," Indian Journal of Chemistry, vol. 41, no. 10, pp. 2145-2149, 2002.

[5] R. Sanjeev and V. Jagannadham, "Substituent effects on the spontaneous cleavage of benzyl-gem-dibromides in aqueous solution," Indian Journal of Chemistry, vol. 41, no. 9, pp. 18411844, 2002.

[6] V. Jagannadham, T. L. Amyes, and J. P. Richard, "Kinetic and thermodynamic stabilities of $\alpha$-oxygen-and $\alpha$-sulfur-stabilized carbocations in solution," Journal of the American Chemical Society, vol. 115, no. 18, pp. 8465-8466, 1993.

[7] T. L. Amyes, J. P. Richard, and V. Jagannadham, "Formation and stability of reactive intermediates of organic reactions in aqueous solution," Royal Society of Chemistry, vol. 148, pp. 334-350, 1995.

[8] R. Sanjeev and V. Jagannadham, "Effect of $\alpha$-halogen atom and $\alpha$-azido group on thermodynamic stability and kinetic reactivity of benzyl carbocations in aqueous solution," Indian Journal of Chemistry, vol. 41, no. 10, pp. 2150-2152, 2002.

[9] J. F. J. Dippy and F. R. Williams, "Chemical constitution and the dissociation constants of monocarboxylic acids. Part II," Journal of the Chemical Society, pp. 1888-1892, 1934.

[10] J. F. J. Dippy, H. B. Watson, and F. R. Williams, "Chemical constitution and the dissociation constants of monocarboxylic acids. Part IV. A discussion of the electrolytic dissociation of substituted benzoic and phenylacetic acids in relation to other side-chain processes," Journal of the Chemical Society, pp. 346350, 1935.
[11] J. F. J. Dippy and R. H. Lewis, "Chemical constitution and the dissociation constants of monocarboxylic acids. Part V. Further substituted benzoic and phenylacetic acids," Journal of the Chemical Society, pp. 644-649, 1936.

[12] The $\mathrm{p} K_{a}$ values of dithio benzoic acids are from a search using SciFinder Scholar software by using the formula index of each acid, Similarly for the thio phenol and 4-nitro thio phenol.

[13] H. H. Jaffé, "A reëxamination of the Hammett equation," Chemical Reviews, vol. 53, no. 2, pp. 191-261, 1953.

[14] V. Jagannadham and S. Steenken, "One-electron reduction of nitrobenzenes by $\alpha$-hydroxyalkyl radicals via addition/elimination. An example of an organic inner-sphere electron-transfer reaction," Journal of the American Chemical Society, vol. 106, no. 22, pp. 6542-6551, 1984.

[15] D. Meisel and P. Neta, "One-electron redox potentials of nitro compounds and radiosensitizers. Correlation with spin densities of their radical anions," Journal of the American Chemical Society, vol. 97, p. 5198, 1975.

[16] P. Neta, M. G. Simic, and M. Z. Hoffman, "Pulse radiolysis and electron spin resonance studies of nitro aromatic radical anions. Optical absorption spectra, kinetics, and one-electron redox potentials," The Journal of Physical Chemistry, vol. 80, p. 2018, 1976. 


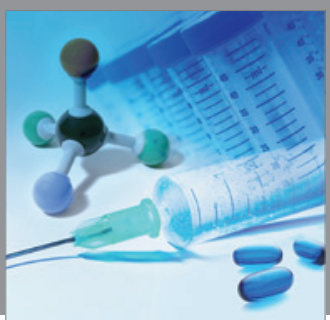

International Journal of

Medicinal Chemistry

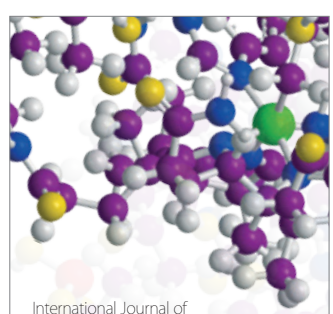

Carbohydrate Chemistry

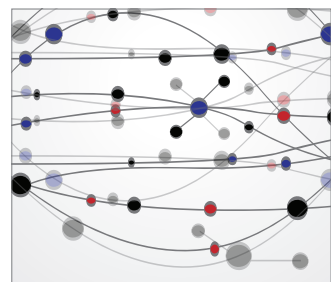

The Scientific World Journal
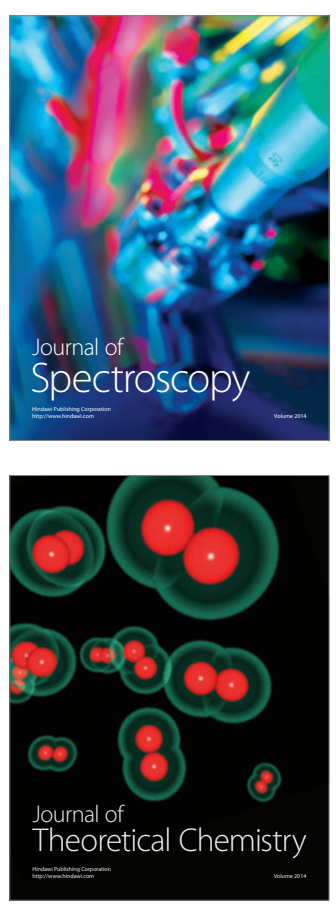
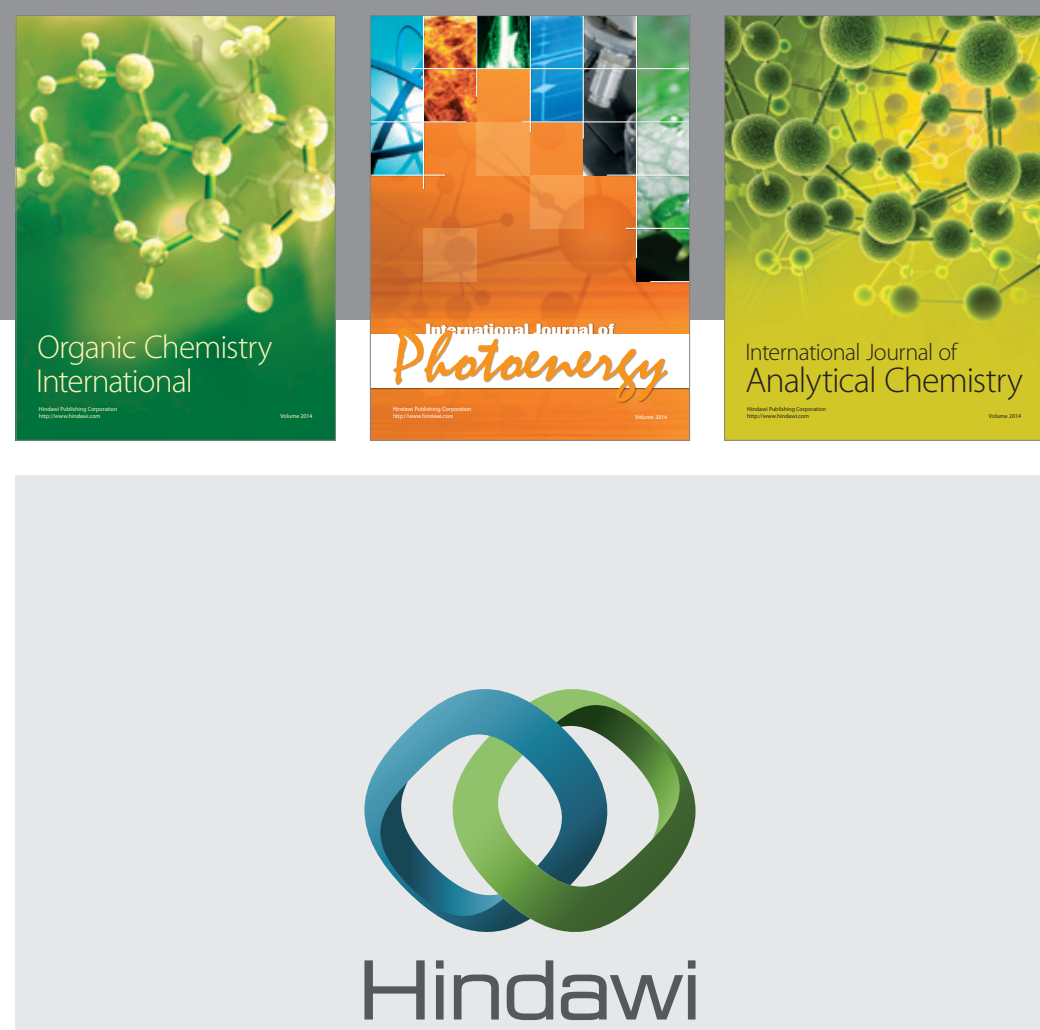

Submit your manuscripts at

http://www.hindawi.com
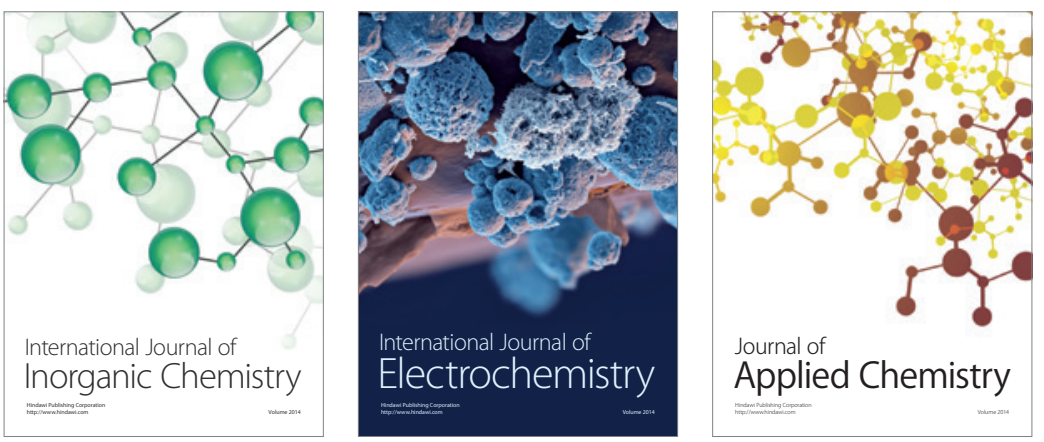

Journal of

Applied Chemistry
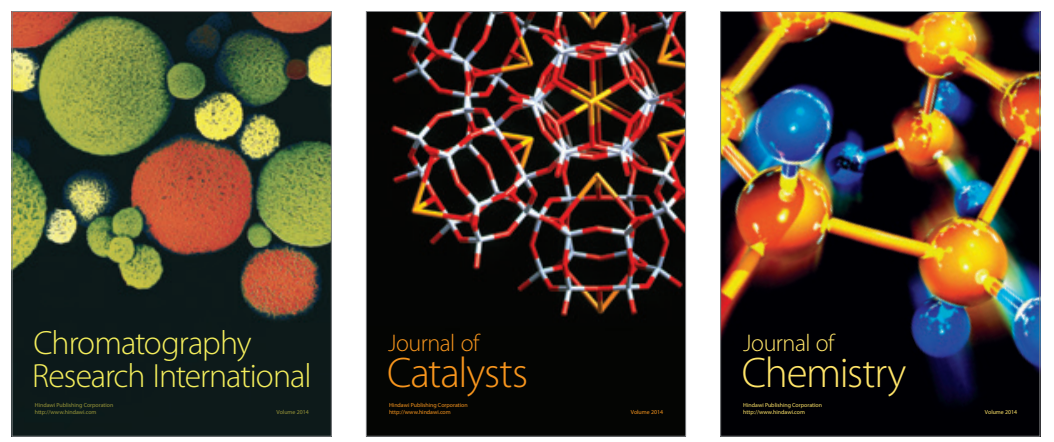
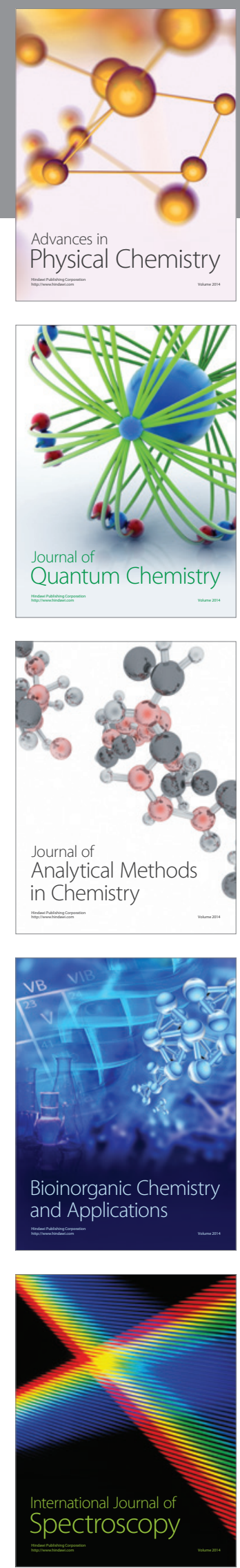